\title{
Efficacy of Prolotherapy with Periarticular 5\% Dextrose in the Treatment of Knee Joint Osteoarthritis
}

\author{
Murat Tolga Avsar 1,2 iD, Resmiye Nur Okudan Kıldan², iD, Zeynep Zehra Gümüs ${ }^{4}$ iD, \\ Rumeysa Samanci ${ }^{5,6}$ \\ Department of Anesthesiology and Reanimation, Gebze Fatih State Hospital, Kocaeli, Turkey \\ 2 Department of Traditional and Complementary Medicine Unit, Gebze Fatih State Hospital, Kocaeli, Turkey \\ ${ }^{3}$ Department of Emergency Medicine, Gebze Fatih State Hospital, Kocaeli, Turkey \\ ${ }^{4}$ Department of Department of Internal Medicine, Izmir Katip Celebi University, Izmir, Turkey \\ 5 Department of Physical Medicine and Rehabilitation, Medicine Faculty, Duzce University, Duzce, Turkey \\ Department of Traditional and Complementary Medicine Center, Duzce University, Duzce, Turkey \\ * Corresponding Author: Murat Tolga Avsar, e-mail: dr.tolgaavsar@gmail.com
}

Received: 03.11.2021

Accepted: 13.12.2021

\begin{abstract}
Objective: Determination of the effectiveness of periarticular 5\% dextrose prolotherapy in the treatment of knee pain due to osteoarthritis.

Material and Methods: The clinical results of prolotherapy with 5\% periarticular dextrose in adult female and male patients with knee pain and complaints related to osteoarthritis were evaluated retrospectively. During the examination, we identified the sensitive ligaments in the knee and injected $1 \mathrm{cc}$ of $5 \%$ dextrose with a $0.6 * 60 \mathrm{~mm}$ sterican needle. In routine practice, stretching exercises are recommended to patients after prolotherapy and for analgesia, a combined analgesic containing paracetemol $500 \mathrm{mg}+$ codeine phosphate $10 \mathrm{mg}+$ caffeine $30 \mathrm{mg}$ is given for the first 3 days. In addition to the initial application, prolotherapy is performed 3 times with an interval of 20 days, and when the Visual Analogue Scale (VAS) score decreases by $80 \%$ or more after the first or second injection, prolotherapy is terminated.

Results: 30 patients aged between 36-70 years were included in the study. Patients' mean age was 52,9 $\pm 9,0$ years. Nineteen patients received 3 sessions and 11 patients received 2 sessions of prolotherapy. A significant decrease was achieved in VAS scores of all patients except one patient. The VAS score was 2.9 at the end of the third session meanwhile the initial VAS average was 8.4. None of the patients had any side effects that would terminate the treatment.

Conclusion: The data obtained in the study show that prolotherapy with periarticular 5\% dextrose is effective in the treatment of knee pain and complaints related to osteoarthritis. However, more comprehensive studies will be guiding the clarification of its place in the osteoarthritis' treatment.
\end{abstract}

Keyword: Prolotherapy, Knee, Osteoarthritis, Periarticular 5\% Dextrose, Visual Analog Scale

\section{INTRODUCTION}

Osteoarthritis is a painful, degenerative disease that causes dysfunction in the joints. Osteoarthritis of the knee is a common, chronic, non-inflammatory arthritis that causes joint pain and disability ${ }^{1}$. Knee osteoarthritis is mostly age-related and approximately $30 \%$ of the population over the age of 65 has osteoarthritis ${ }^{2}$. Knee osteoarthritis is an important public health problem because of its high prevalence, besides causing loss of workforce and expensive treatment methods ${ }^{3}$. The mechanism of pain and functional impairment in osteoarthritis is complex. There are genetic, biochemical, biomechanical and psychosocial factors. The fact that patients respond differently to different treatments, suggests that there is no common pain mechanism in knee osteoarthritis ${ }^{4}$. Although exercise and weight loss are effective in knee osteoarthritis, they are often not sufficient ${ }^{5}$. 


\author{
Volume: 2 Issue: 3 \\ Year: 2021 \\ DOI: $10.53811 /$ ijtcmr. 1018433
}

International Journal of Traditional and Complementary

Medicine Research
Publisher

Duzce University
Complementary treatments such as physiotherapy, analgesic drugs, acupuncture and herbal therapy have also been shown to be effective in patients with knee osteoarthritis ${ }^{6,7}$. Current guidelines suggest that a multidisciplinary approach should be followed in the treatment of knee osteoarthritis, including non-pharmacological, pharmacological, surgical and complementary therapies ${ }^{8}$. In advanced knee osteoarthritis, knee replacement is a highly effective but costly treatment. Therefore, the development of a safe and effective treatment option that complements the current conservative treatment remains a priority in clinical trials ${ }^{9}$.

Prolotherapy is a regenerative and complementary treatment method. With prolotherapy, proliferating substances are applied with a purpose of creating microtraumas in the desired anatomical regions. Different proliferating agents and cells can be applied in this process. For example, dextrose solutions, mannitol, sodium morrhuate, platelet-rich plasma and stem cells are a few of them ${ }^{10,11}$. Dextrose solutions are mostly used as $12.5-25 \%$ periarticular and articular hypertonic solutions. In vitro studies show that dextrose solutions can have similar histological and molecular effects at lower levels ${ }^{12}$. The clinical results of our prolotherapy applications with 5\% dextrose periarticular injections to patients with knee joint osteoarthritis were retrospectively evaluated in this study.

\section{MATERIALS AND METHODS}

We started our study after receiving ethics committee approval from the Gebze State Hospital with protocol number 2019-42. In this study, the records of 30 patients; who applied to the Traditional and Complementary Medicine Polyclinic with knee pain due to osteoarthritis and were treated with $5 \%$ dextrose periarticular prolotherapy; were reviewed and the data obtained were retrospectively evaluated. Informed consent was obtained from each patient.

Our patients were asked to consult their physical therapy and rehabilitation physicians before injection.

\section{Inclusion criteria}

Patients with stage 2 and stage 3 according to the Kellgren Lawrence classification were included in the study. Before the injection, the painful ligaments of the knee were identified and $1 \mathrm{cc}$ of $5 \%$ dextrose was injected into each painful and tender ligament. Patients were monitored during the injection. Saturation values and peak heart rate were followed during the injection and observed after the injection.

\section{The prolotherapy application process}

In our outpatient clinic, prolotherapy is not applied to patients with rheumatological disorders, bleeding disorders, and needle phobia. During this application, it is recommended to do stretching exercises after prolotherapy and an analgesic containing paracetamol $500 \mathrm{mg}+$ codeine phosphate $10 \mathrm{mg}+$ caffeine $30 \mathrm{mg}$ is given for the first 3 days. In our polyclinic, after the initial application of prolotherapy, 3 more sessions are performed with an interval of 20 days.

Pain severity was assessed by Visual Analogue Scale (VAS) scoring at baseline and at each session. If the patient's VAS score decreases $80 \%$ or more after the first or second injection, the treatment is terminated.

\section{Statistical analysis}

SPSS software (IBM SPSS, Version 22.0, IBM Corporation, Armonk, NY, USA) was used for data analysis. Data were presented as mean and standard deviation. T and repetitive ANOVA tests were used to compare VAS scores between sessions. $\mathrm{P}<0.05$ was considered significant.

\section{RESULTS}

30 patients were included in the study in total. Among them, 5 (16.7\%) were male and 25 (83.3\%) were female. The mean age of the patients was 52.9 \pm 9.0 (minimum-maximum values: $36-70$ ).

All patients got 2 sessions of prolotherapy meanwhile only 19 patients got their $3^{\text {rd }}$ session of prolotherapy. The mean VAS scores of the patients, measured at the beginning and during the sessions, are shown in Figure 1.

The mean of VAS score measured during the 1st, 2nd and 3rd session were all significantly lower than the mean of the initial VAS score. $(4.7 \pm 1.5$, $3.1 \pm 1.8$, and $2.9 \pm 1.8$ vs. $8.4 \pm 1.1 ; \mathrm{p}<0.05)$. The mean VAS scores measured during the 2 nd and 3rd sessions were significantly lower than the mean of the VAS scores measured during the 1 st session $(3.1 \pm 1.8$ vs. $2.9 \pm 1.8$ vs. $4.7 \pm 1.5 ; p<0.05)$. The mean VAS scores measured during the 2nd and 3rd session were found to be similar ( $p>0.05)$ (Figure 1). 


\begin{tabular}{|c|c|}
$\begin{array}{c}\text { Volume: } 2 \text { Issue: } 3 \\
\text { Year: } 2021\end{array}$ & $\begin{array}{c}\text { International Journal of Traditional and Complementary } \\
\text { Medicine Research }\end{array}$ \\
DOI: $10.53811 /$ ijtcmr. 1018433 &
\end{tabular}

Publisher
Duzce University

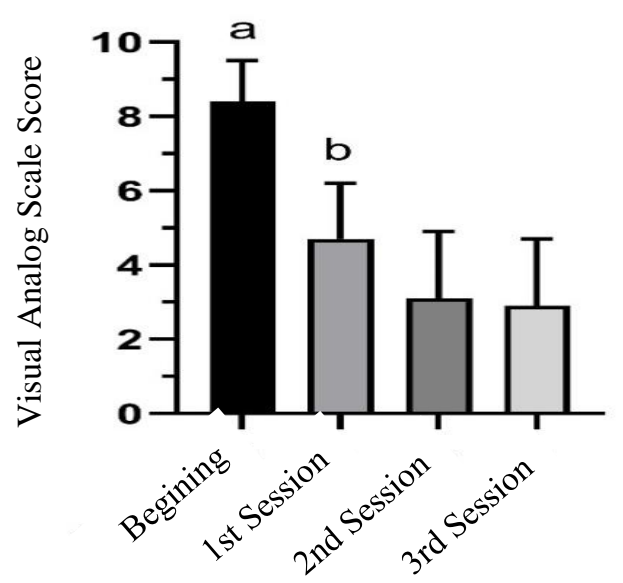

There was no significant difference between the mean VAS scores of male and female patients measured at baseline and between sessions (Figure 2).

\section{DISCUSSION}

In this study, 5\% dextrose periarticular injections of prolotherapy which were applied 3 times with an interval of 3 weeks for the first time, were more successful in patients with knee osteoarthritis especially in female patients. Less post-injection pain at periarticular injection sites increased the patient's satisfaction and adherence to treatment.

Osteoarthritis, the most common form of arthritis, is an important disease of western societies, primarily affecting the knee joint and causing progressive loss of function, pain and stiffness ${ }^{13}$.

Figure 1.Visual analog scale (VAS) scores measured at baseline and in the 1st, 2nd and 3rd sessions.

The VAS scores of male and female patients measured at baseline and during sessions are shown in Figure 2. In male patients, the mean VAS score measured in the 3rd session was lower than that measured at the beginning ( $7 \pm 1.4$ vs. $8.8 \pm 1.3$ ). In women, the mean VAS scores measured in the 1st, 2nd and 3rd sessions were found to be significantly lower than the baseline $(4.6 \pm 1,3.1 \pm 1.3,2.4 \pm 1$ versus $8.4 \pm 1,1 ; \mathrm{p}<0.05)$. The mean VAS scores measured in the 2 nd and 3rd sessions were found to be significantly lower in women compared to the 1 st session ( $3.1 \pm 1.3$ and $2.4 \pm 1$ versus $4.6 \pm 1 ; p<0.05$ ), but the mean VAS scores measured in the 2 nd and 3rd sessions were similar ( $p>0.05$ ).

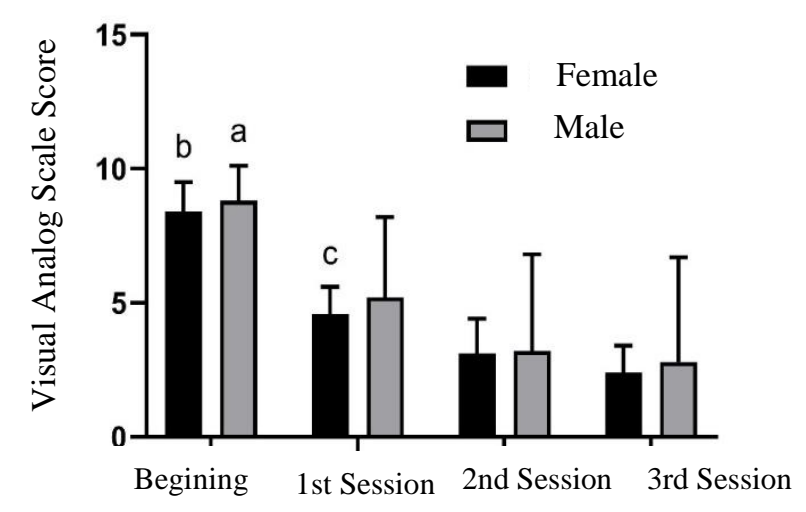

Figure 2. Visual analog scale (VAS) scores of female and male patients measured at the beginning and $1 \mathrm{st}$, 2nd and 3rd sessions.

Osteoarthritis has the potential to worsen progressively by affecting the extra-articular structures as well as the degeneration of intraarticular cartilage, bone and synovial tissues.

Because of the lack of successful and easy-to-apply treatment options for knee joint osteoarthritis, a continued research is required on new treatment applications. Various non-invasive and minimally invasive methods are used in the treatment of pain associated with knee osteoarthritis.

The most common treatments are physical therapy, the use of nonsteroidal anti-inflammatory drugs, and if these methods are ineffective, intra-articular injection of hyaluronic acid, corticosteroid and platelet-rich plasma as well as prolotherapy are used $^{13,14}$.

Factors such as the individual and social burden of osteoarthritis, the damage it causes to work life, and the overuse of health services by the patient can significantly impair the quality of life of individuals. Some important risk factors have been identified for the development of osteoarthritis, which can be seen in $10 \%$ of populations over the age of 50 .

While some of these risk factors are demographic factors such as age, female gender, being overweight and having a family history of osteoarthritis depending on the structural characteristics of the person, many of them may be due to lifelong local and unwanted mechanical traumas that disrupt the joints ${ }^{15} .83 \%$ of the cases included in our study were female.

The risk factors mentioned above and the different pathogenesis of osteoarthritis are important in terms of offering alternative methods such as prolotherapy in addition to the traditional medical and surgical 


\author{
Volume: 2 Issue: 3 \\ Year: 2021 \\ DOI: $10.53811 /$ ijtcmr.1018433
}

International Journal of Traditional and Complementary

Medicine Research
Publisher
Duzce University treatments used in the treatment of this disease.

Prospectively managed research results with new studies in cases of osteoarthritis developed due to different mechanisms will be useful in terms of understanding the clinical efficacy of prolotherapy and in which osteoarthritis types it is more effective ${ }^{13}$. Since $83 \%$ of the cases included in our study were female and the sample size of female was sufficient, the success of prolotherapy was clearly observed in female patients. Due to the small number of male patients (17\%), some of the differences in VAS scores did not reach significance. The mechanism of action of prolotherapy is multifaceted. Fibroblast and vascular proliferation, intense collagen deposition and stimulation of cartilage growth are some of them. In addition, elevated levels of extracellular dextrose hyperpolarize the nerves through a different mechanism. Activation of potassium channels by dextrose leads to increased potassium conductivity and consequent neuronal hyperpolarization. The analgesic effect of hyperpolarization with dextrose injection has been proven. With dextrose prolotherapy, neurogenic inflammation is prevented and thus pain is reduced ${ }^{17,18}$.

In a study comparing periarticular prolotherapy and intraarticular prolotherapy, it was found that the VAS score was lower in the periarticular prolotherapy group. Improvements in walking on level ground, climbing stairs, and morning stiffness were similar in both groups, and positive responses were obtained. Intra-articular injections are used in the treatment of arthritis, but this method is still discussed among many clinicians because of the needle penetration into the joint capsule and possible side effects.

Periarticular injections are more safety than intraarticular injections. It can give similar or even better results ${ }^{19}$. In our study, we performed periarticular dextrose injection and found significant decreases in VAS values.

Seven et al. investigated the efficacy of exercise and ultrasonography-guided prolotherapy in patients with chronic pain associated with a rotator cuff lesion. It has been observed that the success of treatment generally increases with prolotherapy ${ }^{20}$. In our study, we applied 5\% dextrose periarticular prolotherapy and gave stretching exercises to the patients. We believe that prolotherapy combined with exercise therapy will be more effective.

The difference of prolotherapy from other regenerative injection treatments is that it is not injected with biological material as in platelet-rich plasma and stem cell injections. 5\% dextrose and hypertonic glucose are preferred in prolotherapy applications because they are cheap, easily available and safe ${ }^{11}$. For these reasons, we used 5\% dextrose in our study, which is effective, inexpensive, easy to access and has few side effects.

As an alternative method in the treatment of knee osteoarthritis cases, intra-articular prolotherapy with hypertonic glucose is also being investigated increasingly ${ }^{21,22}$.

Sit et al. compared intra-articular hypertonic dextrose prolotherapy administered 4, 8 and 16 weeks after the initial application with normal saline injections over a period of one year ${ }^{21}$.

Rabago et al. tested the efficacy of extra-articular $15 \%$ hypertonic dextrose and intra-articular $25 \%$ dextrose in the treatment of patients with knee osteoarthritis. Prolotherapy has produced beneficial results in terms of pain and mobility in patients with moderate and severe knee osteoarthritis. Our study is on extra-articular prolotherapy and we concluded that prolotherapy is an effective treatment for pain ${ }^{22}$.

This study had some limitations. Relatively lack of number of the patients included in the study, the fact that the disorders in the joints have not been determined objectively, and probably the fact that the studies are conducted in cases with osteoarthritis of different severity require a cautious approach to the statistical results. Functional changes were not examined in our study. This is a limitation of our study. Randomized-controlled studies with more patients are needed in the future.

\section{CONCLUSION}

The result of this study supports that prolotherapy performed with a similar method can be applied as an alternative method in the treatment of knee osteoarthritis. Prolotherapy using periarticular 5\% dextrose is a good option in patients with knee osteoarthritis. Periarticular 5\% dextrose application by prolotherapy specialists in similar clinical situations can positively affect patient satisfaction and treatment success. Keeping detailed clinical information of the patients, performing prolotherapy with a similar protocol to the patients, interpreting the treatment results based on similar and objective criteria and keeping records of possible unexpected situations by physicians who practice prolotherapy in patients with knee osteoarthritis may provide a more established treatment for patients with osteoarthritis. 
Volume: 2 Issue: 3

Year: 2021

DOI: $10.53811 /$ ijtcmr.1018433
International Journal of Traditional and Complementary

Medicine Research
Publisher

Duzce University

\section{REFERENCES}

1. Felson DT. Osteoarthritis of the knee. N Engl J Med. 2006; 354(8):841-8.

2. Woo J, Leung J, Lau E. Prevalence and correlates of musculoskeletal pain in Chineseelderlyandtheimpact on 4-year physical function and quality of life. Public Health. 2009; 123(8):549-56.

3. Sayer EC, Li LC, Kopec JA, Esdaile JM, Cibere J. Theeffect of disease site (knee, hip, hand, foot, lowerbackorneck) on employment due to osteoarthritis. Plos one. 2010; 5(5): e10470

4. Hawker GA, Gignac MA, Badley E. A longitudinal study to explainthe pain-depression link in older adults with osteoarthritis. Arthritis Care \& Res. 2011;63(10):1382-90

5. Hutton I, Gamble G, Mclean G, Butcher H, Gow P, Dalbeth N. Obstaclestoaction in arthritis: a communitycasecontrolstudy. Int J Rheum Dis. 2009; 12(2):107-17.

6. Samson DJ, Grant MD, Ratko TA, Bonnell CJ, Ziegler KM, Aronson N. Treatment of primary and secondar yosteoarthritis of the knee. Evid Rep Technol Assess (Full Rep). 2007; (157):1-157.

7. Recommendations for the medical management of osteoarthritis of the hip and knee: 2000 update. American College of Rheumatology Subcommittee on Osteoarthritis Guidelines. Arthritis Rheum. 2000;43(9):1905-15.

8. Hochberg MC, Altman RD, April KT, Benkhalti M, Guyatt G, McGowan J. AmericanCollege of Rheumatology 2012 recommendations for the use of non pharmacologic and pharmacologic therapies in osteoarthritis of the hand, hip, and knee. Arthritis Care Res (Hoboken). 2012; 64(4):465-74.

9. Felson DT. Osteoarthritis: priorities for osteoarthritis research: much to be done. Nat Rev Rheumatol. 2014; 10(8):447-8.

10. Kim WJ, Shin HY, Koo GH, Park HG, Ha YC, Park YH. Ultrasound-guided Prolotherapy with Polydeoxyribonucleotide Sodium in Ischiofemoral Impingement Syndrome. Pain Pract. 2014; 14(7):649-55.

11. Solmaz İ, Akpancar S, Örsçelik A, Yener-Karasimav Ö, Gül D. Dextrose injections for failed backsurgery syndrome: a consecutive case series. EurSpine J. 2019; 28(7):1610-1617.

12. Hauser RA, Lackner JB, Steilen-Matias D, Harris DK. A SystematicReview of Dextrose Prolotherapy for Chronic Musculoskeletal Pain. Clin Med Insights Arthritis Musculoskelet Disord. 2016; 9:139-59.

13. Rabago D, Nourani B. ProlotherapyforOsteoarthritisandTendinopathy: a DescriptiveReview. Curr Rheumatol Rep. 2017; 19(6):34.

14. Rahimzadeh P, Imani F, Faiz SH, Alebouyeh MR, Azad-Ehyaei D, Bahari L. Adding Intra-Articular Growth Hormone to Platelet Rich Plasma under Ultrasound Guidance in Knee Osteoarthritis: A Comparative Double-Blind Clinical Trial. Anesth Pain Med. 2016; 6(6):e41719.

15. Hussain SM, Neilly DW, Baliga S, Patil S, Meek R. Kneeosteoarthritis: a review of management options. Scott Med J. 2016; 61(1):7-16.

16. Yoshii, Y. Efects of multiple injections of hypertonic dextrose in the rabbit carpal tunnel: a potential model of carpal tunnel syndrome development. Hand (N.Y.) 2014; 9(1):52-7.

17. Reeves KD, Sit RW, Rabago DP. DextroseProlotherapy: A Narrative Review of Basic Science, Clinical Research, and Best Treatment Recommendations. Phys Med Rehabil Clin N Am. 2016; 27(4):783-823.

18. Lyftogt J. Subcutaneous prolotherapy treatment of refractory knee, shoulder and lateral elbow pain. Aust Musculoskeletal Med. 2007; 12(2):110-112.

19. Rezasoltani Z, Taheri M, Mofrad MK, Mohajerani SA. Periarticular dextrose prolotherapy instead of intra Articular injection for pain and functional improvement in knee osteoarthritis. J Pain Res. 2017; 10:1179-1187.

20. Seven MM, Ersen O, Akpancar S, Ozkan H, Turkkan S, Yildız Y. Effectiveness of prolotherapy in the treatment of chronic rotator cuff lesions. Orthop Traumatol Surg Res. 2017; 103(3):427-433.

21. Sit RWS, Wu RWK, Reeves KD, Rabago D, Chan DCC, Yip BHK. Efficacy of intra-articular hypertonic dextrose prolotherapy versus normal saline for knee osteoarthritis: a protocol for a triple-blinded randomized controlled trial. $B M C$ Complement Altern Med. 2018; 18(1):157.

22. Rabago D, Zgierska A, Fortney L, Kijowski R, Mundt M, Ryan M. Hypertonic dextrose injections (prolotherapy) for knee osteoarthritis: results of a single-armun controlled study with 1-year follow-up. J Altern Complement Med. 2012; 18(4):408 